\title{
Analysis of socioeconomic and demographic factors and imaging exam characteristics associated with missed appointments in pediatric radiology
}

\author{
Efrén J. Flores ${ }^{1,2}$ (D) Dania Daye ${ }^{1} \cdot$ Miguel A. Peña ${ }^{1,3} \cdot$ Diego B. Lopez $^{1} \cdot$ Camilo Jaimes $^{2,4} \cdot$ McKinley Glover IV $^{1,2}$
}

Received: 29 December 2020 / Revised: 12 April 2021 / Accepted: 17 May 2021 / Published online: 11 June 2021

(C) The Author(s), under exclusive licence to Springer-Verlag GmbH Germany, part of Springer Nature 2021

\begin{abstract}
Background Missed appointments can have an adverse impact on health outcomes by delaying appropriate imaging, which can be critical in influencing treatment decisions.

Objective To assess for socioeconomic and imaging exam factors associated with missed appointments among children scheduled for diagnostic imaging.

Materials and methods We retrospectively analyzed children ( $<18$ years) scheduled for outpatient diagnostic imaging during a 12 -month period. In doing so, we obtained socioeconomic and radiology exam characteristics (modality, intravenous contrast administration, radiation and use of sedation) data from the electronic medical record. We employed multivariate logistic regression to assess the association of socioeconomic, demographic and imaging exam characteristics with imaging missed appointments.

Results In total, 7,275 children met inclusion criteria. The mean age was 8.8 years (standard deviation [SD] = 6.2 years) and the study population consisted of $52 \%$ female gender, $69 \%$ White race, $38 \%$ adolescent age group and $32 \%$ with a median household income by ZIP-code category of $<\$ 50,000$. Logistic regression showed increased likelihood of missed appointments among children of Black/African-American race (odds ratio $[\mathrm{OR}]=1.9 ; 95 \%$ confidence interval $[\mathrm{CI}]=1.4-2.5$ ); with insurance categories including Medicaid ( $\mathrm{OR}=2.0 ; 95 \% \mathrm{CI}=1.6-2.4)$, self-pay $(\mathrm{OR}=2.1 ; 95 \% \mathrm{CI}=1.3-3.6)$ and other $(\mathrm{OR}=2.7 ; 95 \%$ $\mathrm{CI}=1.3-5.4)$; with $<\$ 50,000$ median household income by ZIP-code category ( $\mathrm{OR}=1.7 ; 95 \% \mathrm{CI}=1.4-2.0)$; and with examination wait time of $7-21$ days $(\mathrm{OR}=2.7 ; 95 \% \mathrm{CI}=2.1-3.5)$ and $>21$ days $(\mathrm{OR}=3.7 ; 95 \% \mathrm{CI}=2.9-4.8)$. The use of radiation, intravenous contrast agent or sedation was not associated with increased likelihood of missed appointments.

Conclusion Expanding our knowledge of how different socioeconomic and imaging-related factors influence missed appointments among children can serve as a foundational step to better understand existing and emerging disparities and inform strategies to advance health equity efforts in radiology.
\end{abstract}

Keywords Children $\cdot$ Health equity $\cdot$ Missed appointment $\cdot$ Missed care opportunity $\cdot$ Pediatric radiology

Efrén J. Flores

ejflores@mgh.harvard.edh

1 Department of Radiology, Massachusetts General Hospital, 55 Fruit St., BLK SB-0029A, Boston, MA 02114, USA

2 Harvard Medical School, Boston, MA, USA

3 Harvard Kennedy School of Government, Cambridge, MA, USA

4 Department of Radiology, Boston Children's Hospital, Boston, MA, USA

\section{Introduction}

Missed appointments can have an adverse impact on health outcomes by delaying appropriate imaging, which can be critical in influencing treatment decisions. Health care organizations colloquially refer to missed appointments as "no-shows." The term "imaging missed care opportunity" has been proposed as a less pejorative term that takes into consideration the influence of social determinants of health in access to medical appointments and the role of the health care system in facilitating medical appointments [1]. Research has demonstrated that socioeconomic and demographic factors as well as increased time from scheduling to 
exam date are predictive of increased imaging missed care opportunities in adults [2,3]. This finding represents an important opportunity for radiologists to engage in assessment and redesign of traditional operations to improve coordination and equitable access to imaging services [2, 3]. Furthermore, imaging missed care opportunities can create significant challenges to health care organizations in terms of access to health services and operations [4-7].

When considering potential causes and opportunities to mitigate the impact of imaging missed care opportunities, special attention is required for pediatric patients. Children undergoing diagnostic imaging are largely influenced by dependence on caregivers for financial considerations, transportation to appointments, and health literacy considerations related to radiation exposure, intravenous contrast use and the use of sedation as part of diagnostic imaging. Because children are in a stage of rapid development, in the long term children stand to gain the most from timely access to health care or conversely suffer the most from the complications of suboptimal care vis-à-vis adults because of children's longer life expectancy. One study of missed appointments for congenital cardiac MRI found that demographic factors were associated with higher rates of missed appointments, although half of patients studied were older than 18 years [8]. This is consistent with similar studies in the outpatient pediatric subspecialty setting that have reported associations between male gender, morning appointment time and public insurance, and higher rates of missed appointments $[9,10]$. Nevertheless, the research on missed appointments within pediatric radiology is limited.

Understanding socioeconomic and imaging factors associated with imaging missed care opportunities in children is important to inform the development of strategies to improve equitable access to pediatric radiology for all children. In previous pediatric literature, the effects of insurance type, imaging modality, the use of sedation and other factors on imaging missed care opportunities have not been comprehensively studied. Therefore, the purpose of this study was to assess whether specific socioeconomic factors and imaging exam characteristics were associated with increased likelihood of missed appointments among children. Our findings expand on current pediatric literature and provide some insight into how these factors impact the odds of experiencing imaging missed care opportunities.

\section{Materials and methods}

\section{Study design and setting}

Our institutional review board approved this single-institution retrospective cohort study, which complied with the Health
Insurance Portability and Accountability Act. Using the institution's radiology information system (RIS; Centricity, version 10.7; GE Healthcare, Chicago, IL), we identified all outpatient diagnostic imaging studies that required an appointment and were scheduled to be performed at a large urban quaternary academic medical center within a 12-month period (April 1, 2015 , to March 30, 2016) for pediatric patients ages 0-17 years. We included outpatient examinations scheduled at the institution's main campus (hospital-based) and affiliated outpatient imaging sites.

\section{Inclusion criteria of diagnostic imaging exams}

All outpatient diagnostic imaging modalities that required a scheduled appointment prior to the diagnostic imaging examination were included: CT, MRI, US, fluoroscopy and nuclear medicine. Radiographs were excluded from the analysis because these do not require a scheduled appointment prior to the examination. All inpatient and emergency department diagnostic imaging and any imaging performed as part of a procedure (e.g., US-guided biopsy) were also excluded from the analysis.

\section{Diagnostic imaging exam characteristics}

Diagnostic imaging examinations were categorized by location if they were performed at the main hospital campus or at one of the affiliated free-standing imaging centers. These exams were also sub-categorized by radiation exposure and relative invasiveness of the exam (e.g., use of intravenous line for contrast administration). These sub-categories included the use of (1) radiation (CT, fluoroscopy, nuclear medicine) or no radiation (US and MRI); (2) intravenous contrast agent (i.e. contrast-enhanced MRI or CT) or no intravenous contrast agent; and (3) sedation or no sedation.

\section{Patient demographic variables}

We queried the institution's research patient data registry to obtain demographic information from the electronic medical record system for all included subjects [11]. Patient ages were stratified into four developmental groups for purposes of analysis: infants $(<2$ years $)$, toddlers ( $3-5$ years $)$, elementary school ages (6-12 years) and adolescents (13-17 years). Additional self-reported demographic data obtained included gender, race/ethnicity, primary language, primary insurance payor and ZIP (zone improvement plan) code of home address. We used the median household income for the patient's ZIP code, as reported in the 2015 Census data [12], as a measure of socioeconomic status (SES). Median household income was stratified into the following groups: $<\$ 50,000$, $\$ 50,000-74,999, \$ 75,000-99,999$ and $\$ 100,000+$. 


\section{Imaging missed care opportunities}

Imaging missed care opportunities have been defined as scheduled imaging appointments that were not performed and not rescheduled prior to their scheduled date and time [2]. The term "imaging missed care opportunities," as opposed to the term "no-shows," reflects the radiologist's role in population health and the multifaceted reasons that result in missed appointments and prevent patients from engaging with their care. Multiple-body-part examinations of the same modality (e.g., CT) scheduled on the same date were classified as one imaging encounter to avoid double-counting and overestimation of imaging missed care opportunities. For example, if a child had been scheduled to have CT examinations of the chest and abdomen/pelvis on the same date and the child did not arrive for the examination, it was deemed to represent one imaging missed care opportunity. However, if a child had been scheduled to have MRI and CT examinations on the same day, the child could have two separate imaging missed care opportunities if he or she did not arrive for either appointment. Encounters were classified as either an imaging missed care opportunity or as a completed encounter and these were mutually exclusive.

\section{Wait days}

Wait days were previously defined as the number of days between the date the examination was ordered within the computerized order entry system and the date the examination was performed or scheduled to be performed (if the appointment was missed) [3]. For example, an examination that was ordered on Jan. 1 and performed Jan. 15 would constitute 14 wait days. Likewise, an examination performed the same day it was ordered constituted 0 wait days. Based on previous analysis, wait days categories were defined as $<7,7-21$ and $>21$ days [3].

Our institution's insurance authorization process is heterogeneous. Authorization is automatic with insurances that have established contracts with our institution and if, at the time of entering the order, it is supported by a clinical decision support system. There are other insurances for which authorization is not automatic but, given our institution's robust support system, we believe that the lack of immediate automaticity has not led to a significant delay in examinations. Accordingly, we are confident that our wait day estimates are not significantly impacted by our heterogeneous insurance authorization processing.

\section{Statistical analyses}

We used descriptive statistics to quantify the total number of outpatient imaging examinations, frequency of demographic characteristics, imaging modalities and total number and frequency of imaging missed care opportunities. Differences in imaging missed care opportunity frequencies by demographic factors, exam characteristics (location, imaging modality, use of radiation, use of intravenous contrast or use of sedation), and wait day categories were assessed using $\chi^{2}$ analyses.

We employed multivariate logistic regression to determine whether race, age category, gender, insurance payor, median household income by ZIP code, wait day category, radiation exposure, use of intravenous contrast agent, or use of sedation was independently associated with at least one imaging missed care opportunity during the study period. To reduce the effect of feature multicollinearity, we performed backward stepwise feature selection (for feature-entry $P=0.05$ was significant and for feature-removal $P=0.10$ was significant using the Wald test). In each model, we assessed significance for each factor using the odds ratio (OR) and the Wald test. All statistical analyses were performed using Stata 14 (StataCorp, College Station, TX).

\section{Results}

A total of 7,275 of outpatient imaging appointments met inclusion criteria. The study population was $52.0 \%(n=3,758)$ female and 8.8 years old on average (standard deviation [SD] $=6.2$ years). The distribution of children based on age categories was $25.3 \%(n=1,844)$ infants, $9.0 \%(n=654)$ toddlers, $287.6 \%(n=2,005)$ elementary school age and $38 \%(n=2,772)$ adolescents. Self-reported racial/ethnic distribution was 69.4\% ( $n=5,048)$ White, $10.3 \%(n=747)$ Hispanic, $5.7 \%$ $(n=412)$ Black/African American and 4.2\% ( $n=304)$ Asian; the rest were "other" $(n=463 / 6.3 \%)$ or "not recorded" $(n=301 / 4.1 \%)$. Using ZIP-code median household income as a proxy for household income, $32.4 \%(2,356 / 7,275)$ of the study population was classified as having an income of $<\$ 50,000 ; 30.3 \%(n=2,205), \$ 50,000-74,999 ; 17.3 \%$ $(n=1,256), \$ 75,000-99,999$; and $20.0 \%(n=1,458)$, $>\$ 100,000$. Additional details of the descriptive statistics of other demographic factors and exam characteristics are included in Table 1.

The overall imaging missed care opportunities rate was $8.2 \%$ $(599 / 7,275)$ over the study period. Statistical $\chi^{2}$ analyses revealed statistically significant differences in imaging missed care opportunity rates by age group category, income category, insurance payor category, self-reported race/ethnicity, selfreported language, wait day category, imaging modality, location where the exam was performed, use of intravenous contrast agent and use of sedation during the exam. Toddlers shared the highest proportion of imaging missed care opportunities, at $12.5 \%$, while adolescents had the lowest share of imaging missed care opportunities at $6.5 \%$. In terms of ethnicity, African Americans shared the highest proportion of imaging 
Table 1 Demographics of study population

\begin{tabular}{|c|c|}
\hline Characteristic & $n(\%)$ \\
\hline \multicolumn{2}{|l|}{ Gender } \\
\hline Female & $3,758(52.0)$ \\
\hline Male & $3,517(48.0)$ \\
\hline Developmental age group, mean $\pm \mathrm{SD}$ (years) & $8.83 \pm 6.2$ \\
\hline Infant $(<2$ years $)$ & $1,844(25.3)$ \\
\hline Toddler ( $2-5$ years $)$ & $654(9.0)$ \\
\hline Elementary (6-12 years) & $2,005(27.6)$ \\
\hline Adolescent (13-17 years) & $2,772(38.1)$ \\
\hline \multicolumn{2}{|l|}{ Race/ethnicity } \\
\hline White & $5,048(69.4)$ \\
\hline Black/African American & $412(5.7)$ \\
\hline Hispanic & $747(10.3)$ \\
\hline Asian & $304(4.2)$ \\
\hline Other & $463(6.3)$ \\
\hline Not recorded or declined & $301(4.1)$ \\
\hline \multicolumn{2}{|l|}{ Primary language } \\
\hline English & $6,373(87.6)$ \\
\hline Spanish & $644(8.9)$ \\
\hline Other & $258(3.5)$ \\
\hline \multicolumn{2}{|l|}{ Insurance type } \\
\hline Commercial & $5,762(79.2)$ \\
\hline Medicaid & $1,282(17.6)$ \\
\hline Self-pay & $175(2.4)$ \\
\hline Other & $56(0.8)$ \\
\hline \multicolumn{2}{|l|}{ Median household income of patient ZIP code } \\
\hline$<\$ 50,000$ & $2,356(32.4)$ \\
\hline$\$ 50,000-74,999$ & $2,205(30.3)$ \\
\hline$\$ 75,000-99,999$ & $1,256(17.3)$ \\
\hline$\$ 100,000+$ & $1,458(20.0)$ \\
\hline \multicolumn{2}{|l|}{ Location of imaging } \\
\hline Main hospital campus & $5,043(69.3)$ \\
\hline Outpatient imaging center & $2,232(30.7)$ \\
\hline \multicolumn{2}{|l|}{ Imaging modality } \\
\hline $\mathrm{CT}$ & $471(6.5)$ \\
\hline MRI & $2,668(36.7)$ \\
\hline Nuclear medicine & $575(7.9)$ \\
\hline Fluoroscopy & $350(4.8)$ \\
\hline Ultrasound & $3,211(44.1)$ \\
\hline Total & $7,275(100 \%)$ \\
\hline
\end{tabular}

$S D$ standard deviation

missed care opportunities, with $18.2 \%$, compared to $6.2 \%$ among Whites. Children whose primary language was Spanish had the highest share of imaging missed care opportunities, at $13.5 \%$, compared to $7.5 \%$ of English-speaking patients. Last, those with Medicaid insurance had the highest share of imaging missed care opportunities, with $15.7 \%$, compared to those with commercial insurance at $6.4 \%$. Additional details are provided in Table 2.

Multivariate logistic regression analysis revealed that the following socioeconomic factors were found to be significantly associated with increased likelihood of imaging missed care opportunities: toddler age $(\mathrm{OR}=1.4)$; Black/African-American race $(\mathrm{OR}=2.0)$; Hispanic language $(\mathrm{OR}=1.4)$; Medicaid $(\mathrm{OR}=1.9)$, self-pay $(\mathrm{OR}=2.1)$ and other insurance categories $(\mathrm{OR}=2.5) ;<\$ 50,000$ median household income by ZIP code $(\mathrm{OR}=1.6)$; fluoroscopy imaging modality $(\mathrm{OR}=2.0)$; and wait time of $7-21$ days $(\mathrm{OR}=2.6)$ and $>21$ days $(\mathrm{OR}=3.6)$. These analyses also revealed that outpatient imaging center category $(\mathrm{OR}=0.8)$ and $\$ 100,000+$ median household income by ZIP code $(O R=0.7)$ were significantly associated with decreased likelihood of imaging missed care opportunities. The unadjusted statistically significant differences between higher imaging missed care opportunities and language, use of intravenous (IV) contrast agents and use of sedation variables were not replicated in the adjusted analysis. After backward feature selection, the following socioeconomic factors remained significantly associated with increased likelihood of imaging missed care opportunities: Black/ African-American race $(\mathrm{OR}=1.9)$; Medicaid $(\mathrm{OR}=2.0)$, self-pay $(\mathrm{OR}=2.1)$ and other insurance categories $(\mathrm{OR}=2.7) ;<\$ 50,000$ median household income by ZIP code $(\mathrm{OR}=1.7)$; fluoroscopy imaging modality $(\mathrm{OR}=1.5)$; and wait time of $7-21$ days $(O R=2.7)$ and $>21$ days $(\mathrm{OR}=3.7)$. This analysis also demonstrated that outpatient imaging center $(\mathrm{OR}=0.7)$ and $\$ 100,000+$ median household income by ZIP code $(\mathrm{OR}=0.7)$ categories were associated with decreased likelihood of imaging missed care opportunities. Additionally, MR imaging modality became associated with significantly decreased likelihood of imaging missed care opportunities $(\mathrm{OR}=0.4)$. Additional details are provided in Table 3.

\section{Discussion}

The study results showed an increased likelihood of imaging missed care opportunities among children of Black/AfricanAmerican race, children with Medicaid, self-pay and other insurance categories; fluoroscopy modality; children living in ZIP codes associated with $<\$ 50,000$ median household income; and children waiting 7-21 days and $>21$ days for their scheduled examination. The study results also showed that the use of radiation, intravenous contrast agent or sedation for scheduled diagnostic imaging examination was not associated with increased likelihood for pediatric imaging missed care opportunities. Missed appointments in diagnostic radiology represent a window to understand factors that influence access to diagnostic imaging services among children. Several 
Table 2 Chi-square analyses of factors associated with pediatric imaging missed care opportunities ${ }^{\mathrm{a}}$

\begin{tabular}{|c|c|c|c|}
\hline Characteristics & Completed encounters ( $\%$ of row) & Imaging missed care opportunities ( $\%$ of row) & $P$-value \\
\hline Developmental age group ${ }^{\mathrm{b}}$ & & & $<0.001$ \\
\hline Infant $(<2$ years $)$ & $1,673(90.7)$ & $171(9.3)$ & \\
\hline Toddler $(3-5$ years $)$ & $572(87.5)$ & $82(12.5)$ & \\
\hline Elementary (6-12 years) & $1,838(91.7)$ & $167(8.3)$ & \\
\hline Adolescent (13-17 years) & $2,593(93.5)$ & $179(6.5)$ & \\
\hline Race/ethnicity ${ }^{\mathrm{b}}$ & & & $<0.001$ \\
\hline White & $4,737(93.8)$ & $311(6.2)$ & \\
\hline Black/African American & $337(81.8)$ & $75(18.2)$ & \\
\hline Hispanic & $641(85.8)$ & $106(14.2)$ & \\
\hline Asian & $281(92.4)$ & $23(7.6)$ & \\
\hline Other & $406(87.7)$ & $57(12.3)$ & \\
\hline Not recorded or declined & $274(92.3)$ & $117(7.7)$ & \\
\hline Primary language $\mathrm{e}^{\mathrm{b}}$ & & & $<0.001$ \\
\hline English & $5,894(92.5)$ & $479(7.5)$ & \\
\hline Spanish & $557(86.5)$ & $87(13.5)$ & \\
\hline Other & $225(87.2)$ & $33(12.8)$ & \\
\hline Insurance type ${ }^{b}$ & & & $<0.001$ \\
\hline Commercial & $5,393(93.6)$ & $369(6.4)$ & \\
\hline Medicaid & $1,081(84.3)$ & $201(15.7)$ & \\
\hline Self-pay & $157(89.7)$ & $18(10.3)$ & \\
\hline Other & $45(80.4)$ & $11(19.6)$ & \\
\hline Median household income of patient ZIP code ${ }^{\mathrm{b}}$ & & & $<0.001$ \\
\hline$<\$ 50,000$ & $2,056(86.6)$ & $318(13.4)$ & \\
\hline$\$ 50,000-74,999$ & $2,038(93.2)$ & $149(6.8)$ & \\
\hline$\$ 75,000-99,999$ & $1,180(93.9)$ & $76(6.1)$ & \\
\hline$\$ 100,000+$ & $1,402(96.2)$ & $56(3.8)$ & \\
\hline Location of imaging ${ }^{\mathrm{b}}$ & & & $<0.001$ \\
\hline Main hospital campus & $4,547(90.2)$ & $496(9.8)$ & \\
\hline Free-standing imaging center & $2,129(95.4)$ & $103(4.6)$ & \\
\hline Imaging modality ${ }^{\mathrm{b}}$ & & & $<0.001$ \\
\hline $\mathrm{CT}$ & $439(93.2)$ & $32(6.8)$ & \\
\hline MRI & $2,557(95.8)$ & $111(4.2)$ & \\
\hline Nuclear medicine & $524(91.1)$ & $51(8.9)$ & \\
\hline Fluoroscopy & $312(89.1)$ & $38(10.9)$ & \\
\hline Ultrasound & $2,844(88.6)$ & $367(11.4)$ & \\
\hline Wait days ${ }^{\mathrm{b}}$ & & & $<0.001$ \\
\hline$<7$ & $2,507(96.3)$ & $95(3.7)$ & \\
\hline $7-21$ & $2,058(90.4)$ & $218(9.6)$ & \\
\hline$>21$ & $2,111(88.0)$ & $286(12.0)$ & \\
\hline Radiation use during exams & & & 0.60 \\
\hline Without radiation & $5,386(91.8)$ & $478(8.2)$ & \\
\hline With radiation & $1,290(91.4)$ & $121(8.6)$ & \\
\hline Sedation use during exams ${ }^{\mathrm{c}}$ & & & 0.02 \\
\hline Without sedation & $5,976(91.5)$ & $554(8.5)$ & \\
\hline With sedation & $700(94.0)$ & $45(6.0)$ & \\
\hline IV contrast use during exams ${ }^{\mathrm{c}}$ & & & 0.03 \\
\hline Without contrast agent & $4,588(91.3)$ & $438(8.7)$ & \\
\hline With contrast agent & $2,088(92.8)$ & $161(7.2)$ & \\
\hline
\end{tabular}

IV intravenous

${ }^{a}$ We used Pearson's chi-square tests to assess whether any observed differences in imaging missed care opportunities between different categories for each factor of interest arose by chance

${ }^{\mathrm{b}} P<0.001$ is significant

${ }^{\mathrm{c}} P<0.05$ is significant

important conclusions can be drawn from the results of this study.

Children of lower socioeconomic status (SES) are at increased likelihood of imaging missed care opportunities, a correlation that is consistent with previous studies $[2,6,13]$. Lower SES encompasses several social determinants of health including lower health literacy levels, financial concerns with health-related costs and decreased access to transportation, among many other barriers that predominantly affect financially vulnerable communities [14]. Parents of lower SES might have increased concerns about out-of-pocket costs and high deductibles [15]. To address concerns related to financial 
Table 3 Multivariate logistic regression of factors associated with pediatric imaging missed care opportunities

\begin{tabular}{|c|c|c|c|c|c|c|}
\hline \multirow[t]{2}{*}{ Characteristics } & \multicolumn{3}{|c|}{ Initial multivariate model } & \multicolumn{3}{|c|}{ Model with feature selection } \\
\hline & OR & $95 \% \mathrm{CI}$ & $P$-value & OR & $95 \% \mathrm{CI}$ & $P$-value \\
\hline \multicolumn{7}{|l|}{ Developmental age group } \\
\hline Infant $(<2$ years $)$ & 1.0 & & & & & \\
\hline Toddler ( $3-5$ years $)$ & $1.4^{\mathrm{a}}$ & $1.1-1.9$ & 0.02 & & & \\
\hline Elementary school (6-12 years) & 1.2 & $1.0-1.6$ & 0.08 & & & \\
\hline Adolescent ( $>12$ years) & 1.2 & $0.9-1.5$ & 0.19 & & & \\
\hline \multicolumn{7}{|l|}{ Race/ethnicity } \\
\hline White & 1.0 & & & & & \\
\hline Black/African American & $2.0^{\mathrm{b}}$ & $1.5-2.7$ & $<0.001$ & $1.9^{\mathrm{b}}$ & $1.4-2.5$ & $<0.001$ \\
\hline Hispanic & $1.4^{\mathrm{a}}$ & $1.1-1.9$ & 0.02 & & & \\
\hline Asian & 1.1 & $0.7-1.8$ & 0.63 & & & \\
\hline Other & 1.2 & $0.9-1.7$ & 0.26 & & & \\
\hline Not recorded or declined & 1.3 & $0.8-2.0$ & 0.28 & & & \\
\hline \multicolumn{7}{|l|}{ Primary language } \\
\hline English & 1.0 & & & & & \\
\hline Spanish & 1.08 & $0.7-1.6$ & 0.73 & & & \\
\hline Other & 0.82 & $0.6-1.1$ & 0.20 & & & \\
\hline \multicolumn{7}{|l|}{ Insurance type } \\
\hline Commercial & 1.0 & & & & & \\
\hline Medicaid & $1.9^{\mathrm{b}}$ & $1.5-2.3$ & $<0.001$ & $2.0^{\mathrm{b}}$ & $1.6-2.4$ & $<0.001$ \\
\hline Self-pay & $2.1^{\mathrm{c}}$ & $1.2-3.5$ & 0.007 & $2.1^{\mathrm{c}}$ & $1.3-3.6$ & 0.004 \\
\hline Other & $2.5^{\mathrm{a}}$ & $1.2-5.2$ & 0.01 & $2.7^{\mathrm{c}}$ & $1.3-5.4$ & 0.007 \\
\hline \multicolumn{7}{|c|}{ Median household income of patient ZIP code } \\
\hline$\$ 50,000-74,999$ & 1.0 & & & & & \\
\hline$\$ 75,000-99,999$ & 1.0 & $0.8-1.4$ & 0.95 & $1.3^{\mathrm{b}}$ & $1.2-1.4$ & $<0.001$ \\
\hline$<\$ 50,000$ & $1.6^{\mathrm{b}}$ & $1.3-2.0$ & $<0.001$ & $1.7^{\mathrm{b}}$ & $1.4-2.0$ & $<0.001$ \\
\hline$\$ 100,000+$ & $0.7^{\mathrm{a}}$ & $0.5-1.0$ & 0.03 & $0.7^{\mathrm{a}}$ & $0.5-0.9$ & 0.01 \\
\hline \multicolumn{7}{|l|}{ Site of imaging } \\
\hline Main hospital campus & 1.0 & & & & & \\
\hline Free-standing imaging center & $0.8^{\mathrm{a}}$ & $0.6-1.0$ & $0.02^{\mathrm{a}}$ & $0.7^{\mathrm{c}}$ & $0.6-0.9$ & 0.003 \\
\hline \multicolumn{7}{|l|}{ Imaging modality } \\
\hline CT & 1.0 & & & & & \\
\hline MRI & 0.2 & $0.0-3.0$ & 0.26 & $0.4^{\mathrm{b}}$ & $0.3-0.5$ & $<0.001$ \\
\hline Nuclear medicine & 1.4 & $0.9-2.2$ & 0.19 & & & \\
\hline Fluoroscopy & $2.0^{\mathrm{a}}$ & $1.2-3.3$ & 0.01 & $1.5^{\mathrm{a}}$ & $1.1-2.2$ & 0.03 \\
\hline Ultrasound & 0.8 & $0.1-10.2$ & 0.83 & & & \\
\hline \multicolumn{7}{|l|}{ Wait days } \\
\hline$<7$ & 1.0 & & & & & \\
\hline $7-21$ & $2.6^{\mathrm{b}}$ & $2.0-3.4$ & $<0.001$ & $2.7^{\mathrm{b}}$ & $2.1-3.5$ & $<0.001$ \\
\hline$>21$ & $3.6^{\mathrm{b}}$ & $2.8-4.6$ & $<0.001$ & $3.7^{\mathrm{b}}$ & $2.9-4.8$ & $<0.001$ \\
\hline \multicolumn{7}{|l|}{ Radiation use during exams } \\
\hline Without radiation & 1.0 & & & & & \\
\hline With radiation & 0.5 & $0.3-6.0$ & 0.55 & & & \\
\hline \multicolumn{7}{|l|}{ Sedation use during exams } \\
\hline Without sedation & 1.0 & & & & & \\
\hline With sedation & 1.1 & $0.8-1.7$ & 0.55 & & & \\
\hline \multicolumn{7}{|l|}{ IV contrast use during exams } \\
\hline Without contrast & 1.0 & & & & & \\
\hline With contrast & 1.2 & $0.9-1.7$ & 0.17 & & & \\
\hline
\end{tabular}

$C I$ confidence interval, $O R$ odds ratio, ZIP Zone Improvement Plan

${ }^{\text {a }} P<0.05$ is significant

${ }^{\mathrm{b}} P<0.001$ is significant

${ }^{\mathrm{c}} P<0.01$ is significant

barriers, radiology practices could proactively increase cost transparency and ask caregivers if they have cost concerns related to undergoing diagnostic imaging that might prevent them from pursuing care for their children [16, 17]. Radiology practices could collaborate with health care institutions that help children and their caregivers access financial resources. This might include online portals to governmental programs or private groups that offer support, such as Aunt Bertha, for assistance with other socioeconomic factors that influence access to care [18]. 
The results of our study also showed that children who experience increased wait days between the ordering date and the exam date are at greater likelihood of imaging missed care opportunities, which is consistent with previous research on adults [6,19-23]. Studies have shown that potential reasons for this relationship in the pediatric population include additional scheduling conflicts that arise between patients or caregivers and the health care institution where they receive care [24]. Increasing scheduling flexibility by the health care institution, such as increasing capacity and availability of high-value imaging services, has been shown to decrease missed appointments $[25,26]$. Decreasing wait days to imaging services has also been shown to decrease health disparities among vulnerable populations in radiology [27, 28]. Radiology departments might consider the value and practicality of having additional routine weekend and evening availability for diagnostic imaging services and improved carecoordination with other medical appointments. Such interventions could assist vulnerable patient populations that face many challenges navigating the complex health care system [28].

The imaging missed care opportunity literature on adults has estimated an imaging missed care opportunity rate ranging from $2.0 \%$ to $6.5 \%$ depending on imaging modality $[13,29]$. In our study, we found an overall $8 \%$ rate of imaging missed care opportunities. This rate is larger than such in the adult literature. We attribute this difference to the reality that pediatric patients are exclusively dependent on the availability of their parents and their schedule to bring them to imaging appointments. Thus, potential scheduling conflicts among caregivers with their institution's appointment scheduling system leads to a higher rate of imaging missed care opportunities. The results of this study also showed that the toddler developmental age group was associated with increased likelihood of missed appointments. At our institution, some MRI studies in this age group require the use of conscious sedation for undergoing imaging studies. This invariably comes with an increase in wait days because sedation appointments are scarce; sedation examinations also add to the potential financial burden to the caregiver because they require longer time for preparation and recovery and are also more costly [30]. In addition to using advanced imaging techniques to decrease image acquisition times without the use of sedation, the utilization of child life specialists in pediatric radiology practices has been shown to be an effective resource to assist children and their caregivers [31]. Child life specialists' role is to educate children and their caregivers about their upcoming appointment and to enhance their comfort by addressing the family's concerns [32-34]. Radiology practices can continue to collaborate with referring providers, community health care workers and child life specialists to help alleviate the uncertainty or potential concerns related to their scheduled diagnostic imaging appointment.

Our study also showed that imaging studies requiring the use of radiation, intravenous contrast agent or sedation were not associated with an increased likelihood of missed appointments. Furthermore, MRI, which does not use radiation, was associated with decreased likelihood of imaging missed care opportunities. Although this might be reflective of different reminder processes associated with each imaging modality, radiology has also made substantial efforts through national organizations to increase the availability of information about imaging exams through campaigns such as Image Wisely, Image Gently and Radiologyinfo.org [35-37]. The extent to which children and their caregivers have been informed about what to expect about their diagnostic radiology examination is uncertain because some information portals are written in a way that is more complex than the average reading ability of the population $[37,38]$. However, these continued efforts to increase the availability of multilingual educational material that can be tailored based on developmental age groups and health literacy levels are vital to increase accessibility of health information. Moreover, we found that US had a higher share of imaging missed care opportunities than MRI appointments. This finding is surprising because MRI is usually associated with higher wait days [3], which, as we have shown, is also associated with increased odds of imaging missed care opportunities. Although not examined in our study, this discrepancy might be a result of greater scheduling flexibility inherent with US appointments when compared to MRIs. Therefore, parents might be more willing to miss or reschedule US appointments than MRI appointments based on this flexibility. This discrepancy could also reflect institutional differences of our department's appointment reminder processes vis-à-vis different imaging modalities. Given that MRI appointments are less readily available and take longer to perform, these have additional support and follow-up reminder processes. Further research should examine the impact that the heterogeneity of the appointment reminder process and its interaction with imaging modality have on imaging missed care opportunities.

Our results are consistent with literature regarding missed appointments for well-child visits. It has been shown that among pediatric patients, those who are uninsured, those in a low-income family, and those who are African American are more likely to miss a greater proportion of well-child visits when compared to their high-income, privately insured and White counterparts [39]. Radiology departments should adopt strategies currently used to reduce missed care opportunities for well-child visits. This non-exhaustive list of strategies includes educating the patient's caregivers about the benefits of completed appointments, documenting the reasons why a child did not show up for an appointment, tracking 
missed care opportunities, updating the child's caregiver contact information at every visit, and providing walk-in slots to accommodate children with high rates of missed care opportunities [40].

Expanding our knowledge of how socioeconomic and imaging-related factors influence access to care among children can serve as a foundational step to better understand existing and emerging disparities and advance health equity efforts. The coronavirus disease of 2019 (COVID19) pandemic has brought preexisting health disparities to the fore, underscoring the importance of understanding how social determinants of health directly influence health outcomes among vulnerable populations. Recent literature has shown that individuals living in ZIP codes associated with lower income and higher proportion of limited English proficiency bear a disproportionate burden of COVID-19, including worse outcomes and higher mortality [41-44]. Radiology is strategically positioned to form multidisciplinary collaborations to gain a deep understanding of existing barriers and inform the development of programs to address existing and emerging health disparities among vulnerable communities.

There are several limitations to our study. First, we did not capture whether any of the missed appointments were performed at other institutions or as part of a hospital admission. Second, this single-institution study might not be generalizable to other populations with a higher proportion of diverse racial and ethnic composition, as well as the influence of statefunded children's health insurance, which varies widely by state. We note that our insurance payor mix is different from the nation's average payor mix for pediatric patients - approximately $80 \%$ of our patients have commercial insurance and $17.9 \%$ Medicaid, versus the national average of $53 \%$ commercial and $34 \%$ Medicaid payors [45]. Consequently, our study might overrepresent children with more favorable socioeconomic conditions than those among other pediatric radiology departments. However, this difference does not limit the internal validity of our results regarding the estimated effects of multiple socioeconomic factors on the odds of imaging missed care opportunities. We expect that our results will have the greatest external validity among pediatric radiology departments that have a comparable demographic and socioeconomic composition to ours. Third, the study used median household income based on ZIP code, which is an imprecise proxy for socioeconomic status and might not reflect the individual economic resources of each patient. Fourth, the study did not account for logistical factors to access care such as transportation, patients changing providers of care, insurance pre-authorization processes and differences in appointment reminder processes, among others [3]. For example, MRI appointments that require sedation entail multiple reminders from the institution, which differs from a diagnostic US reminder process. Fifth, the study did not capture supplementary health insurance or differences in out-ofpocket deductible costs associated with different types of insurance, which have been shown to play an important role in the decision to delay or forgo care [46]. Sixth, we recognize that our study does not capture the impact that a heterogeneous insurance pre-authorization process can have in modulating the odds of imaging missed care opportunities. We expect that radiology departments with a more integrated and comprehensive insurance pre-authorization system have a smaller number of wait days and thus lower odds of an imaging missed care opportunity. Nonetheless, the impact of diverse insurance authorization processes on imaging missed care opportunities should be examined in future studies. Seventh, we recognize that other factors might modulate the odds of imaging missed care opportunities that our study did not explore. These include appointment time of day, day of the week (including weekday versus weekend) and history of imaging missed care opportunities. Further research should examine the impact these other factors can have on imaging missed care opportunities. We also recognize that the data derived in this study span the period of 2015-2016. However, we have little reason to believe that our results lack external validity to the present, particularly once outpatient pediatric imaging utilization returns to pre-pandemic levels. Given the lack of a coordinated nationwide effort among pediatric radiology departments in addressing imaging missed care opportunities, we are confident that our results are still relevant and valid today. Nevertheless, some institutions and pediatric radiology departments have made significant improvements at reducing imaging missed care opportunities via pilot programs encouraging price transparency, transportation support and the implementation of a more effective reminder processes. Last, future studies should evaluate the downstream effects of missed appointments in the overall health outcomes of children, and the effect that cost transparency programs can have in decreasing missed appointments.

\section{Conclusion}

This study expands our knowledge of how different socioeconomic and imaging-related factors influence missed appointments among children scheduled to undergo diagnostic imaging and can serve as a foundational step to better understand existing and emerging disparities and inform strategies to advance health equity efforts in radiology.

\section{Declarations}

Conflicts of interest None 


\section{References}

1. Commission on Social Determinants of Health (2008) Closing the gap in a generation: health equity through action on the social determinants of health. Final report of the commission on social determinants of health. World Health Organization, Geneva

2. Glover M, Daye D, Khalilzadeh O et al (2017) Socioeconomic and demographic predictors of missed opportunities to provide advanced imaging services. J Am Coll Radiol 14:1403-1411

3. Daye D, Carrodeguas E, Glover MT et al (2018) Impact of delayed time to advanced imaging on missed appointments across different demographic and socioeconomic factors. J Am Coll Radiol 15: 713-720

4. Satiani B, Miller S, Patel D (2009) No-show rates in the vascular laboratory: analysis and possible solutions. J Vasc Interv Radiol 20 : $87-91$

5. Norbash A, Yucel K, Yuh W et al (2016) Effect of team training on improving MRI study completion rates and no-show rates. J Magn Reson Imaging 44:1040-1047

6. Cronin PR, DeCoste L, Kimball AB (2013) A multivariate analysis of dermatology missed appointment predictors. JAMA Dermatol 149:1435-1437

7. Dantas LF, Fleck JL, Cyrino Oliveira FL, Hamacher S (2018) Noshows in appointment scheduling - a systematic literature review. Health Policy 122:412-421

8. Lu JC, Lowery R, Yu S et al (2017) Predictors of missed appointments in patients referred for congenital or pediatric cardiac magnetic resonance. Pediatr Radiol 47:911-916

9. Huang Z, Ashraf M, Gordish-Dressman H, Mudd P (2017) The financial impact of clinic no-show rates in an academic pediatric otolaryngology practice. Am J Otolaryngol 38:127-129

10. Perez FD, Xie J, Sin A et al (2014) Characteristics and direct costs of academic pediatric subspecialty outpatient no-show events. J Healthc Qual 36:32-42

11. Nalichowski R, Keogh D, Chueh HC, Murphy SN (2006) Calculating the benefits of a research patient data repository. AMIA Annu Symp Proc 2006:1044

12. Posey KB (2016) Household income: 2015. United States Census Bureau website. https://www.census.gov/library/publications/ 2016/acs/acsbr15-02.html. Accessed 10 May 2021

13. Harvey HB, Liu C, Ai J et al (2017) Predicting no-shows in radiology using regression modeling of data available in the electronic medical record. J Am Coll Radiol 14:1303-1309

14. Samuels RC, Ward VL, Melvin P et al (2015) Missed appointments: factors contributing to high no-show rates in an urban pediatrics primary care clinic. Clin Pediatr 54:976-982

15. Wu S, Sylwestrzak G, Shah C, DeVries A (2014) Price transparency for MRIs increased use of less costly providers and triggered provider competition. Health Aff 33:1391-1398

16. Farrell KS, Finocchio LJ, Trivedi AN, Mehrotra A (2010) Does price transparency legislation allow the uninsured to shop for care? J Gen Intern Med 25:110-114

17. De Brantes F, Delbanco S (2016) Report card on state price transparency laws - July 2016. The Heartland Institute website. https:// www.heartland.org/publications-resources/publications/reportcard-on-state-price-transparency-laws-july-2016? source $=$ policybot. Accessed 10 May 2021

18. Aunt Bertha (2019) findhelp.org free search tool. findhelp.org/?ref= ab_redirect. 10 May 2021

19. Shrestha MP, Hu C, Taleban S (2017) Appointment wait time, primary care provider status, and patient demographics are associated with nonattendance at outpatient gastroenterology clinic. J Clin Gastroenterol 51:433-438
20. Chang JT, Sewell JL, Day LW (2015) Prevalence and predictors of patient no-shows to outpatient endoscopic procedures scheduled with anesthesia. BMC Gastroenterol 15:123

21. Kalb LG, Freedman B, Foster C et al (2012) Determinants of appointment absenteeism at an outpatient pediatric autism clinic. $\mathrm{J}$ Dev Behav Pediatr 33:685-697

22. Kogan-Liberman D, Rivas Y, Thompson J, Tomer G (2015) Improving nonattendance at outpatient pediatric endoscopy unit of a tertiary center. J Pediatr Gastroenterol Nutr 61:234-237

23. Whittle J, Schectman G, Lu N et al (2008) Relationship of scheduling interval to missed and cancelled clinic appointments. J Ambul Care Manage 31:290-302

24. Schneiderman JU, Kennedy AK, Sayegh CS (2017) Qualitative study of foster caregivers' views on adherence to pediatric appointments. J Pediatr Health Care 31:104-110

25. Specht EM, Powell KR, Dormo CA (2004) Factors affecting missed appointment rates for pediatric patients insured by Medicaid in a traditional hospital-based resident clinic and hospital-owned practice settings. Clin Pediatr 43:749-752

26. Iben P, Kanellis MJ, Warren J (2000) Appointment-keeping behavior of Medicaid-enrolled pediatric dental patients in eastern Iowa. Pediatr Dent 22:325-329

27. Dontchos BN, Narayan A, Seidler M et al (2019) Impact of a sameday breast biopsy program on disparities in time to biopsy. J Am Coll Radiol 37

28. Wang GX, Pizzi BT, Miles RC et al (2020) Implementation and utilization of a "pink card" walk-in screening mammography program integrated with physician visits. J Am Coll Radiol 17:16021608

29. Rosenbaum JI, Meloszyk RJ, Hall CS et al (2018) Understanding why patients no-show: observations of 2.9 million outpatient imaging visits over 16 years. J Am Coll Radiol 15:944-950

30. Vanderby SA, Babyn PS, Carter MW et al (2010) Effect of anesthesia and sedation on pediatric MR imaging patient flow. Radiology 256:229-237

31. Kozak BM, Jaimes C, Kirsh J, Gee MS (2020) MRI techniques to decrease imaging times in children. Radiographics 40:485-502

32. McGee K (2003) The role of a child life specialist in a pediatric radiology department. Pediatr Radiol 33:467-474

33. Stevenson MD, Bivins CM, O'Brien K et al (2005) Child life intervention during angiocatheter insertion in the pediatric emergency department. Pediatr Emerg Care 21:712-718

34. Rahi JS, Manaras I, Tuomainen H, Hundt G (2004) Meeting the needs of parents around the time of diagnosis of disability among their children: evaluation of a novel program for information, support, and liaison by key workers. Pediatrics 114:e477-e482

35. Frush DP, Strauss KJ (2017) Image Gently: getting it right. J Am Coll Radiol 14:575-576

36. Mayo-Smith WW, Morin RL (2017) Image Wisely: the beginning, current status, and future opportunities. J Am Coll Radiol 14:442443

37. Rubin GD, Krishnaraj A, Mahesh M et al (2017) Enhancing public access to relevant and valued medical information: fresh directions for radiologyinfo.org. J Am Coll Radiol 14:697-702

38. Berland GK, Elliott MN, Morales LS et al (2001) Health information on the Internet: accessibility, quality, and readability in English and Spanish. JAMA 285:2612-2621

39. Wolf ER, O'Neil J, Pecsok J et al (2020) Caregiver and clinician perspectives on missed well-child visits. Ann Fam Med 18:30-34

40. Partners for Kids, Nationwide Children's Hospital (2018) Reducing no-show appointments in pediatric patients. Online document. http://partnersforkids.org/wp-content/uploads/2018/08/W33247 How-to-Reduce-No-Show-Appointment-Fact-Sheet-2.pdf. Accessed 10 May 2021

41. Hooper WM, Nápoles AM, Pérez-Stable EJ (2020) COVID-19 and racial/ethnic disparities. JAMA 323:2466-2467 
42. Ravi K (2020) No ethnic disparities in COVID-19 mortality: are comorbidities to blame? Lancet 396:22

43. Raifman MA (2020) Disparities in the population at risk of severe illness from COVID-19 by race/ethnicity and income. Am J Prev Med 59:137-139

44. Azar KMJ, Shen Z, Romanelli R et al (2020) Disparities in outcomes among COVID-19 patients in a large health care system in California. Health Aff 39:1253-1262

45. Gillis KD (2017) Policy research perspectives: physicians' patient mix - a snapshot from the 2016 Benchmark Survey and changes associated with the ACA. American Medical Association. Online document. https://www.ama-assn.org/sites/ama-assn.org/files/corp/ media-browser/public/health-policy/PRP-2017-physicianbenchmark-survey-patient-mix.pdf. Accessed 10 May 2021

46. Brook RH, Ware JE, Rogers WH et al (1984) The effect of coinsurance on the health of adults: results from the RAND health insurance experiment. The Rand Corp, Santa Monica

Publisher's note Springer Nature remains neutral with regard to jurisdictional claims in published maps and institutional affiliations. 